\title{
Top Quark Production and Properties
}

\author{
Cecilia E. Gerber \\ University of Illinois at Chicago - Department of Physics \\ 845 W. Taylor St. M/C 273, Chicago IL 60607-7059, USA
}

\begin{abstract}
I present recent results on top quark production and properties in $p \bar{p}$ collisions at a center of mass energy of $1.96 \mathrm{TeV}$. The measurements were performed by the CDF and D0 collaborations using approximately $1 \mathrm{fb}^{-1}$ of data taken during Run II at the Tevatron.
\end{abstract}

\section{Introduction}

The top quark was discovered at the Fermilab Tevatron Collider in $1995[2,3]$ and completes the quark sector of the three-generation structure of the standard model (SM). It is the heaviest known elementary particle with a mass approximately 40 times larger than that of the next heaviest quark, the bottom quark. It differs from the other quarks not only by its much larger mass, but also by its lifetime which is too short to build hadronic bound states. The top quark is one of the least-studied components of the SM, and the Tevatron, with a center of mass energy of $\sqrt{s}=1.96 \mathrm{TeV}$, is at present the only accelerator where it can be produced. The top quark plays an important role in the discovery of new particles, as the Higgs boson coupling to the top quark is stronger than to all other fermions. Understanding the production properties of top quark pairs is in itself a test of perturbative Quantum Chromo Dynamics (pQCD); in addition, it is a crucial ingredient in the discovery of new physics beyond the SM.

In the following sections I will present results for the measurement of the $t \bar{t}$ pair production cross section, studies of $t \bar{t}$ pair production mechanisms, and measurements of the top quark charge.

\section{Studies of $t \bar{t}$ pair production mechanisms}

At Tevatron energies, top quarks are produced predominantly in pairs. Within the SM, the top quark decays almost exclusively into a $W$ boson and a $b$ quark, resulting in two $W$ bosons and $2 b$ jets in each $t \bar{t}$ pair event. The $W$ boson itself decays into one lepton and its associated neutrino, or hadronically. We have classified the $t \bar{t}$ pair decay channels as follows: the dilepton channels where both $W$ bosons decay leptonically into an electron or a muon $(e e, \mu \mu, e \mu)$, the lepton + jets channels where one of the $W$ bosons decays leptonically and the other hadronically $(e+$ jets, $\mu+$ jets $)$, and the all-jets channel where both $W$ bosons decay hadronically. Production cross sections have been measured in all decay channels. The lepton + jets channels have less statistics than the all-jets channel, but the background level is significantly smaller, making it the channel of choice for the measurement of top quark properties.

\subsection{Measurement of the $t \bar{t}$ pair production cross section}

The total top quark pair production cross section for a hard scattering process initiated by a $p \bar{p}$ collision at $\sqrt{s}$ is a function of the top quark mass $m_{t}$. For a top quark mass 
of $175 \mathrm{GeV}$, the predicted SM $t \bar{t}$ production cross section is $6.7_{-0.9}^{+0.7} \mathrm{pb}$ [4]. Deviations of the measured cross section from the theoretical prediction could indicate effects beyond QCD perturbation theory. Explanations might include substantial non-perturbative effects, new production mechanisms, or additional top quark decay modes beyond the SM. Previous measurements $[5,6]$ show good agreement with the theoretical expectation within the experimental precision.

Recent new results became available from both CDF and D0 based on approximately $1 \mathrm{fb}^{-1}$ of data. They are summarized in Fig. 1, together with the theoretical predictions. As can be seen in the plots, the uncertainties on the latest experimental results are reaching the theoretical uncertainty of $\approx 12 \%$, with $10 \%$ uncertainty expected once the $2 \mathrm{fb}^{-1}$ of data already on tape are analyzed.
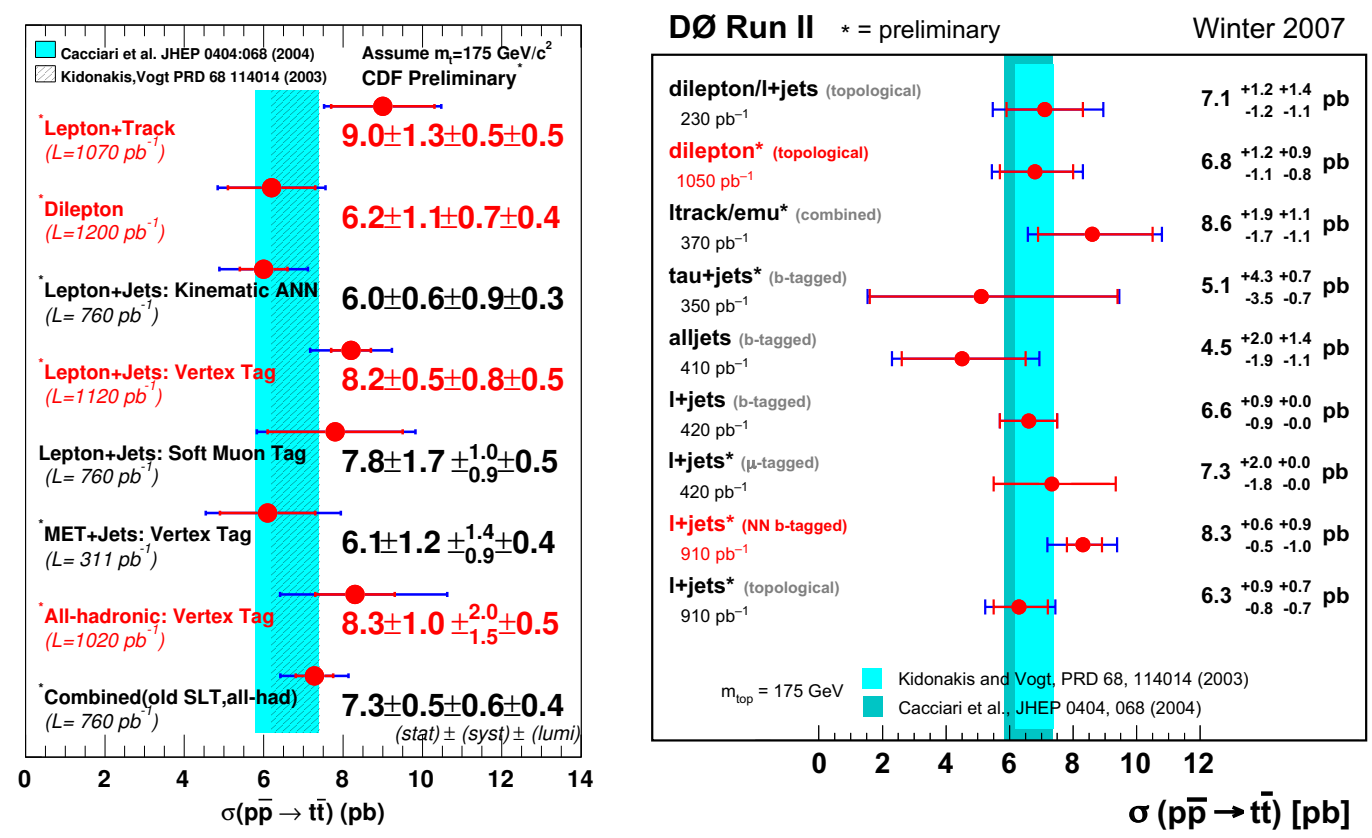

Figure 1: Summary of $t \bar{t}$ pair production cross section measurements at $\sqrt{s}=1.96 \mathrm{TeV}$ from CDF (left), and D0 (right). Theoretical predictions are shown as vertical bands. The newest experimental results have total uncertainties (excluding luminosity) of $\approx 15 \%$, close to the theoretical uncertainty of $12 \%$.

\subsection{Measurement of $\frac{\sigma(g g \rightarrow t \bar{t})}{\sigma(q \bar{q} \rightarrow t \bar{t})}$}

At leading order, $t \bar{t}$ production proceeds through the $q \bar{q} \rightarrow t \bar{t}$ and $g g \rightarrow t \bar{t}$ processes, with the $q \bar{q}$ process contributing $85 \%$ to the production cross section, and the $g g$ process contributing only $15 \%$. NLO theoretical predictions are available [4], but suffer from large uncertainties. Measuring the relative fraction of $t \bar{t}$ events produced via a particular production mechanism provides a direct test of $\mathrm{pQCD}$ and may reveal the existence of $t \bar{t}$ production and decay mechanisms beyond the ones predicted by the SM [7]. 
CDF has studied the relative fraction of $t \bar{t}$ events produced via gluon-fusion: $\frac{\sigma(g g \rightarrow t \bar{t})}{\sigma(q \bar{q} \rightarrow t \bar{t})}$. Two methods were used for this analysis. The first one takes advantage of the fact that the top quark decays before hadronizing, allowing the different production processes to retain their kinematic characteristics in the final state. A neural network was built using two production and six decay variables and was used to maximize the discrimination between $g g$ and $q \bar{q}$ produced events. Using $695 \mathrm{pb}^{-1}$ of data, a $95 \%$ C.L. limit on the relative fraction of $t \bar{t}$ events produced by the $g g$ process is found to be less than $51 \%$.

The second method uses the clear correlation observed between the average number of gluons and the average number of low $p_{T}$ charged particles present in a given sample to extract the fraction of $t \bar{t}$ events that originate from the $g g$ process. Using $1 \mathrm{fb}^{-1}$ of data, CDF measures for the first time $\frac{\sigma(g g \rightarrow t \bar{t})}{\sigma(q \bar{q} \rightarrow t \bar{t})}=0.01 \pm 0.16$ (stat) \pm 0.07 (syst), in agreement with SM expectations.

\subsection{Search for $t \bar{t}$ Resonances}

Several beyond the SM theories [8] predict the resonant production of $t \bar{t}$ pairs. Using $955 \mathrm{pb}^{-1}$ of data, CDF has studied the $t \bar{t}$ invariant mass spectrum in lepton + jets events containing at least 4 jets, with at least one jet being identified as originating from a $b$ quark. The sample contains 347 events, with $73 \pm 9$ events expected to originate from background processes. The observed spectrum is consistent with SM expectations, showing no evidence for additional resonant production mechanisms. This agreement is used to set various model-dependent limits on resonant $t \bar{t}$ production [9].

\section{Measurement of the top quark charge}

The electric charge, one of the fundamental properties of particles, has not been determined yet for the top quark. Within the SM, the top quark has charge $q=+2 e / 3$ and decays into a $W^{+}$boson and a bottom quark $(q=-1 e / 3)$. An alternative scenario [10] has been proposed, in which the top quark observed at the Tevatron would actually be an exotic quark of charge $q=-4 e / 3$, that decays into a $W^{-}$boson and a bottom quark. Within this exotic model (XM), the SM top quark would be more massive, and not observed yet. The first method to discriminate between the two hypotheses was developed by the D0 collaboration. Using $370 \mathrm{pb}^{-1}$ of integrated luminosity, D0 found that their data agreed much better with the SM than with the XM hypothesis [11].

A new analysis [12] recently became available by the CDF collaboration based on $695 \mathrm{pb}^{-1}$ of lepton + jets events with at least 2 jets identified as $b$-jets, and $955 \mathrm{pb}^{-1}$ of dilepton events with at least $1 b$-jet. The charge of the top quark is determined from the charge of the decay products. The charge of the $W$ boson is directly taken from the charge of the lepton. The charge of the jets identified as $b$-jets is determined by combining the $p_{T}$ and charge of the tracks associated to the jet. This procedure assigns the correct charge to the jet in approximately $60 \%$ of the cases. The last step is the association of the lepton and the $b$-jet originating from the same top decay branch. The association is estimated from MC to be correct in $86 \%$ of the cases for the lepton + jets events, and in $96 \%$ of the cases for the dilepton events. The result is extracted using the technique of hypothesis testing. CDF uses the SM as the null hypothesis, and selects, a-priori, the probability of incorrectly rejecting the SM to be $1 \%$. If the XM is true, $81 \%$ of all p-values will fall below 0.01 . The measured p-value from data under the SM hypothesis is 0.35 . This value is greater than $1 \%$, meaning 
that the XM hypothesis is excluded at the $81 \%$ C.L. CDF also computes the Bayes Factor and concludes that the data strongly favor the SM over the XM hypothesis.

\section{Conclusions}

The Tevatron has entered a new era of top quark precision measurements. The experimental precision on the top quark pair production cross section results is approaching the theoretical uncertainty, making comparisons between different channels and methods interesting. In addition, a series of new measurements of top quark properties are becoming available based on the larger statistics samples collected in $1 \mathrm{fb}^{-1}$ of collider data. All measurements are in agreement with SM expectations.

CDF and D0 have already written to tape more than twice the amount of data used for these results. The lepton + jets sample with two identified $b$-jets is completely dominated by $t \bar{t}$ events. With larger data sets, as the ones that will be available at the Tevatron in the near future, this sample will allow for precise measurements of top quark properties for the first time.

\section{Acknowledgments}

I would like to thank my collaborators from the CDF and D0 collaborations for their help in preparing this document. I also thank the staffs at Fermilab and collaborating institutions, and acknowledge support from the National Science Foundation (USA).

\section{References}

[1] Slides: http://indico.cern. ch/contributionDisplay.py? contribId=106\&sessionId=9\&conf Id=9499

[2] F. Abe et al., CDF Collaboration, Phys. Rev. Lett. 742626 (1995).

[3] S. Abachi et al., D0 Collaboration, Phys. Rev. Lett. 742632 (1995).

[4] N. Kidonakis and R. Vogt, Phys. Rev. D 68, 114014 (2003). M. Cacciari, S. Frixione, M. L. Mangano, P. Nason, and G. Ridolfi, JHEP 0404, 68 (2004).

[5] D. Acosta et al., CDF Collaboration, Phys. Rev. Lett. 93, 142001 (2004); Phys. Rev. D 71, 052003 (2005); Phys. Rev. D 71, 072005 (2005); Phys. Rev. D 72, 032008 (2005); Phys. Rev. D 74, 072005 (2006); Phys. Rev. Lett. 96, 202002 (2006); Phys. Rev. Lett. 97, 082004 (2006).

[6] V. M. Abazov et al., Do Collaboration, Phys. Lett. B 626, 35 (2005); Phys. Lett. B 626, 45 (2005); Phys. Lett. B 626, 55 (2005); Phys. Rev. D 74, 112004 (2006).

[7] G.L. Kane and S. Mrenna, Phys. Rev. Lett. 77, 3502-3505 (1996).

[8] A. Leike, Phys. Rep. 317, 143 (1999); J. Rosner, CERN-TH/96-169 (1996); T. Rizzo, Phys. Rev. D 61, 055005 (2000); L. Sehgal, M. Wanninger, Phys. Lett. B 200, 211 (1988); C. Hill, S. Park, Phys. Rev. D 49, 4454 (1994).

[9] CDF Collaboration public result: http://www-cdf.fnal.gov/physics/new/top/2006/mass/mttb/mttb_pub.pdf

[10] D. Chang, W. Chang, and E. Ma, Phys. Rev. D 59, 091503 (1999); 61, 037301 (2000); D. Choudhury, T.M. Tait, and C. E. Wagner, Phys. Rev. D 65, 053002 (2002).

[11] V. M. Abazov et al., D0 Collaboration, Phys. Rev. Lett. 98, 041801 (2007).

[12] CDF Collaboration public result:

http://www-cdf.fnal.gov/physics/new/top/2007/topProp/top_charge/publicTQpage.html 